\title{
Perhydropyrimidinylium and 1,3-diazepinylium salts as potential ionic liquids
}

\author{
Barbara Zaleska ${ }^{*},{ }^{1}$, Marcin Karelus ${ }^{1}$, Michał Flasiński, ${ }^{1}$ Paweł Serda ${ }^{2}$ \\ Department of Organic Chemistry ${ }^{\prime}$, Jagiellonian University, Regional Laboratory of \\ Physicochemical Analysis and Structural Research ${ }^{2}$, ul. Ingardena 3, PL 30-060 Kraków, \\ Poland \\ E-mail: zaleska@,chemia.uj.edu.pl
}

\section{Dedicated to Professor Jan Epsztajn on His $75^{\text {th }}$ birthday}

\begin{abstract}
Saturated or partially saturated heterocyclic compounds were uncovered as interesting and potential ionic liquid media. Zwitterionic compounds with perhydropyrimidinylium, 1,3diazepinylium and quinazolinylium carbocations were utilized in the preparation of a variety of salts in order to search for new low-melting ionic media. Some of the zwitterionic quinazoline derivatives were also found to be low-melting glasses.
\end{abstract}

Keywords: Ionic liquids, perhydropyrimidine, diazepine, quinazoline

\section{Introduction}

Ionic liquids have become very popular in recent years and have proven to be versatile alternatives to classic synthetic procedures using common organic solvents. Application in organic synthesis is enormous and still increasing. They have become of great importance in development of laboratory or industrial catalytic processes ${ }^{1-4}$ and biocatalysis. ${ }^{5,6}$ They have been used in various polymerization processes and preparation of ion conductive polymer electrolytes. ${ }^{7,8}$ Technical applications of ionic liquids present big diversity including preparation of photosensitizers, ${ }^{9}$ solar cells, ${ }^{10,11}$ lubricants for steel, ${ }^{12,13}$ improvement of aerogels production $^{14}$ and HPLC methods, ${ }^{15}$ and even embalming and tissue preservation for biological purposes. $^{16}$

We have already investigated 2-anilino-2-methoxy-3-oxothiobutanoic acid anilides 1a-c in the synthesis of various heterocyclic systems ${ }^{17,18}$ and found unusual rearrangement leading to water soluble zwitterionic compounds 2, 3 with perhydropyrimidine and 1,3-diazepine moiety 
(Scheme 1) when 1,3- and 1,4-diamine were used. The structures of these compounds were confirmed by X-ray analysis of $2 .^{17}$

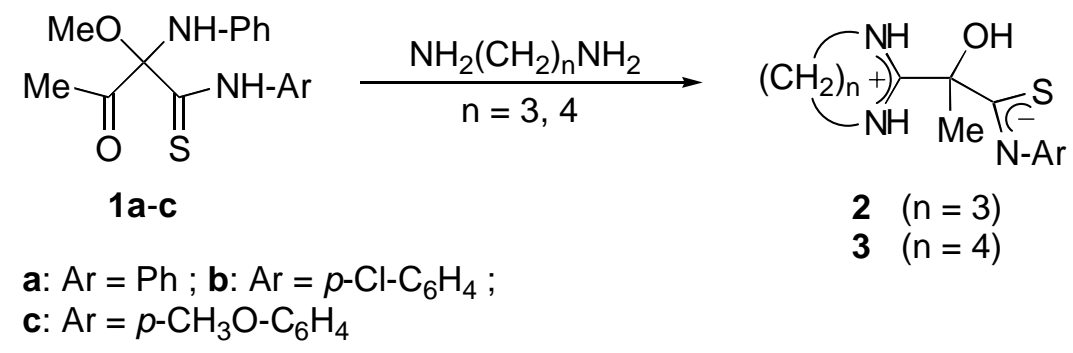

\section{Scheme 1}

We have also found that zwitterions $\mathbf{2}$ and $\mathbf{3}$ may form yellow crystalline products when treated with inorganic acids and this was attributed to the quantitative formation of salts with a thioamide group in the cationic moiety (Scheme 2).

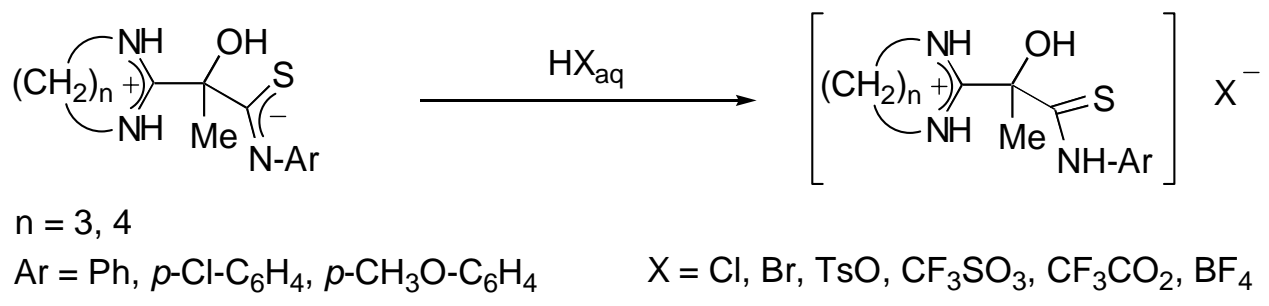

\section{Scheme 2}

This fact brought us the idea to investigate whether salts of this type could be liquid at low temperatures and offer a novel reaction medium for further research.

\section{Results and Discussion}

In our studies we used zwitterionic compounds $\mathbf{2 , 3}$ and zwitterions with various modifications in the perhydropyrimidine ring 4-8 (Figure 1). Zwitterionic compounds 4 with quinazolidinylium moiety are interesting examples of low-melting glasses resembling natural resins and they are liquid above the room temperature. 

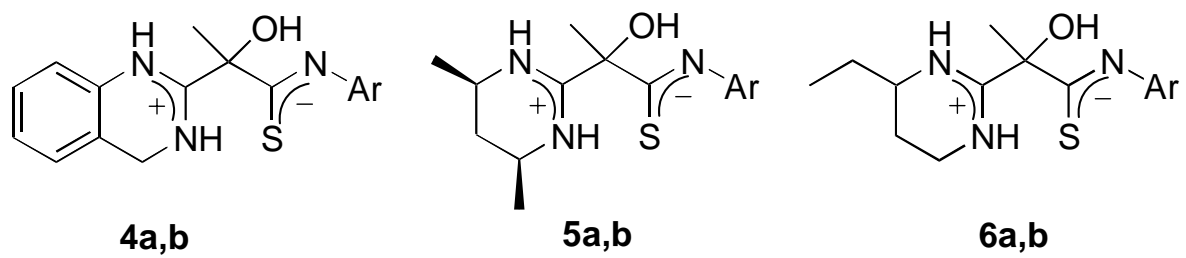<smiles>CC(O)(C(=S)Nc1ccccc1)C1=NCC(O)CN1</smiles>

7<smiles>CC1(C)CN=C(C(C)(O)C(=S)Nc2ccccc2)NC1</smiles>

8

$$
\mathrm{Ar}=\mathbf{a}: \mathrm{Ph}, \mathbf{b}: p-\mathrm{Cl}-\mathrm{C}_{6} \mathrm{H}_{4}
$$

\section{Figure 1}

All of them were prepared according to the reaction in Scheme 1 and were converted into their salts by dissolving appropriate zwitterionic compound 2-8 in dilute aqueous solutions of the corresponding acid. Hydrochloric, hydrobromic, trifluoromethanesulfonic, $p$-toluenesulfonic, trifluoroacetic, and tetrafluoroboric acids were used. After evaporation to dryness under reduced pressure, yellow products were dried in vacuum at elevated temperature. All products were characterized by their NMR spectra and elemental analyses, which confirmed the assumed structure and showed that the products were very pure and did not require further treatment. High-melting salts could, however, be easily crystallized from acetonitrile or toluene-acetonitrile mixture.

Changes in NMR spectra of a substrate and its salt can be explained on the example of a representative pair 2 and 2' (Figure 2) and these considerations can be extended for all other pairs of compounds.

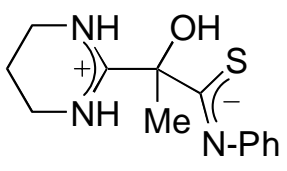

2

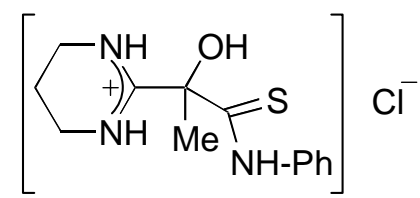

2'

\section{Figure 2}

${ }^{1} \mathrm{H}$ NMR spectrum of $\mathbf{2}^{\prime}$ ' shows singlet at $\delta=11.57 \mathrm{ppm}$, which is not present in the spectrum of the substrate $\mathbf{2}$ and is characteristic for the proton of the thioamide group. Three groups of signals from protons of perhydropyrimidine ring in the substrate: triplet at $\delta=3.47$, quintet at $\delta=$ $1.93 \mathrm{ppm}$ and the broad signal at $\delta=8.0-9.5 \mathrm{ppm}$ ( $\mathrm{NH}$ protons) appear in the ${ }^{1} \mathrm{H} \mathrm{NMR}$ spectrum of the product at $\delta=3.37, \delta=1.81$ and $\delta=9.53 \mathrm{ppm}$, respectively, which proves that 
the cationic perhydropyrimidine moiety remains unchanged. In ${ }^{13} \mathrm{C}$ NMR spectra of 2 and $\mathbf{2}^{\prime}$ characteristic changes are observed in the position of the signal from the benzene ring carbon atom attached to the nitrogen atom. Value of $\delta=151.7 \mathrm{ppm}$ in the spectrum of $\mathbf{2}$ indicates the presence of $\mathrm{N}$-phenylimine form with a negative charge located on the sulfur atom while the value of $\delta=137.3 \mathrm{ppm}$ in the spectrum of $\mathbf{2}^{\prime}$ is characteristic for a neighboring ordinary thioamide group. The ${ }^{13} \mathrm{C}$ NMR spectrum of the product $\mathbf{2}^{2}$ also shows a typical signal of the thioamide carbon atom at $\delta=198.7 \mathrm{ppm}$.

Anion type appears to have no significant effect on the chemical shifts of ${ }^{1} \mathrm{H}$ and ${ }^{13} \mathrm{C}$ NMR signals. IR spectra are not particularly diagnostic and MS spectra for salts were not recorded, since they present the same fragmentation as for the starting compounds. X-ray analysis of the hydrochloride of $\mathbf{4 a}$ was made as an additional proof of the salt structure (Figure 3).

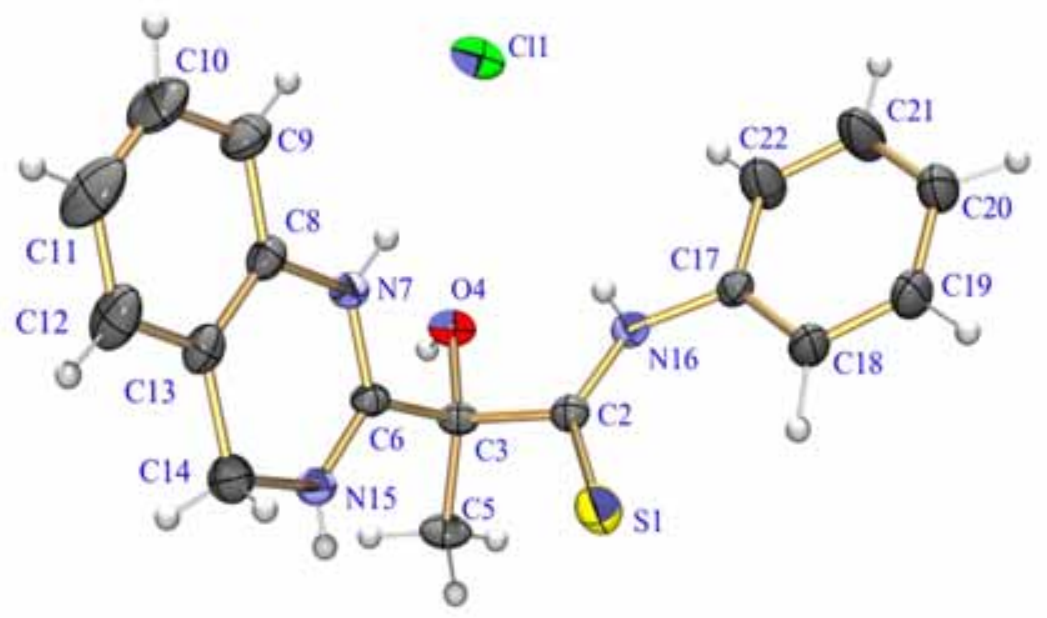

Figure 3. A perspective view of $\mathbf{4} \mathbf{a}^{\prime}$ - protonation product of $\mathbf{4 a}$.

The saturated heteroring C6-N7-C8-C13-C14-N15 has flat conformation. The overall conformation of the molecule is stabilised by several intramolecular close contacts, of which the

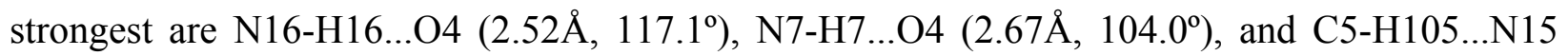
$\left(2.84 \AA, 86.3^{\circ}\right)$, where $\mathrm{H} 105$ is one of the methyl group protons. The chlorine atom is involved in two hydrogen bonds N15-H15...Cl1 $\left(3.11 \AA, 158.7^{\circ}\right)$ and O4-H4...Cl1 $\left(3.06 \AA, 173.4^{\circ}\right)$.

Compound $4 \mathbf{a}^{\prime}$ with formula $\mathrm{C}_{17} \mathrm{H}_{20} \mathrm{~N}_{3} \mathrm{OSCl}$ crystallizes in the monoclinic system, space group $\mathrm{Cc}$, with unit cell parameters $\mathrm{a}=22.5220(5), \mathrm{b}=5.9770(2), \mathrm{c}=12.8750(3) \AA, \beta=$ 93.327(2) $, \mathrm{V}=1730.2(1) \AA^{3}, \mathrm{Z}=4$. A total of 2549 independent reflections $(R($ int $)=0.018)$ were collected on a crystal (size $0.35 \times 0.25 \times 0.15 \mathrm{~mm}$ ) using KappaCCD diffractometer and MoK $\alpha$ radiation. The structure was solved by direct methods with SHELXS $97^{19}$ and refined by the fullmatrix least-squares method on $F^{2}$ using SHELXL9 $7^{20}$ program. Final $R$ indices for $I>2 \sigma(I)$ were equal $R 1=0.0306, \mathrm{w} R 2=0.0752$ and $R 1=0.0337, \mathrm{w} R 2=0.0777$ for all 2549 reflections. The final difference Fourier map of electron density was featureless with the largest peak and hole of 
0.271 and -0.225 e. $\AA^{-3}$, respectively. All calculations and molecular graphics were done using the WinGX package. ${ }^{21}$ The structural data were deposited at the Cambridge Crystallographic Data Centre. These data can be obtained free of charge via www.ccdc.cam.ac.uk/conts/retrieving.html (or from the CCDC, 12 Union Road, Cambridge CB2 1EZ, UK; fax: +44 1223 336033; e-mail: deposit@ccdc.cam.ac.uk) under reference number CCDC 616203.

In the large group of salts synthesized we found some which are low-melting and remain liquid in a broad range of temperatures (Table 1).

Table 1. Melting points and range of the stable liquid phase of low-melting salts

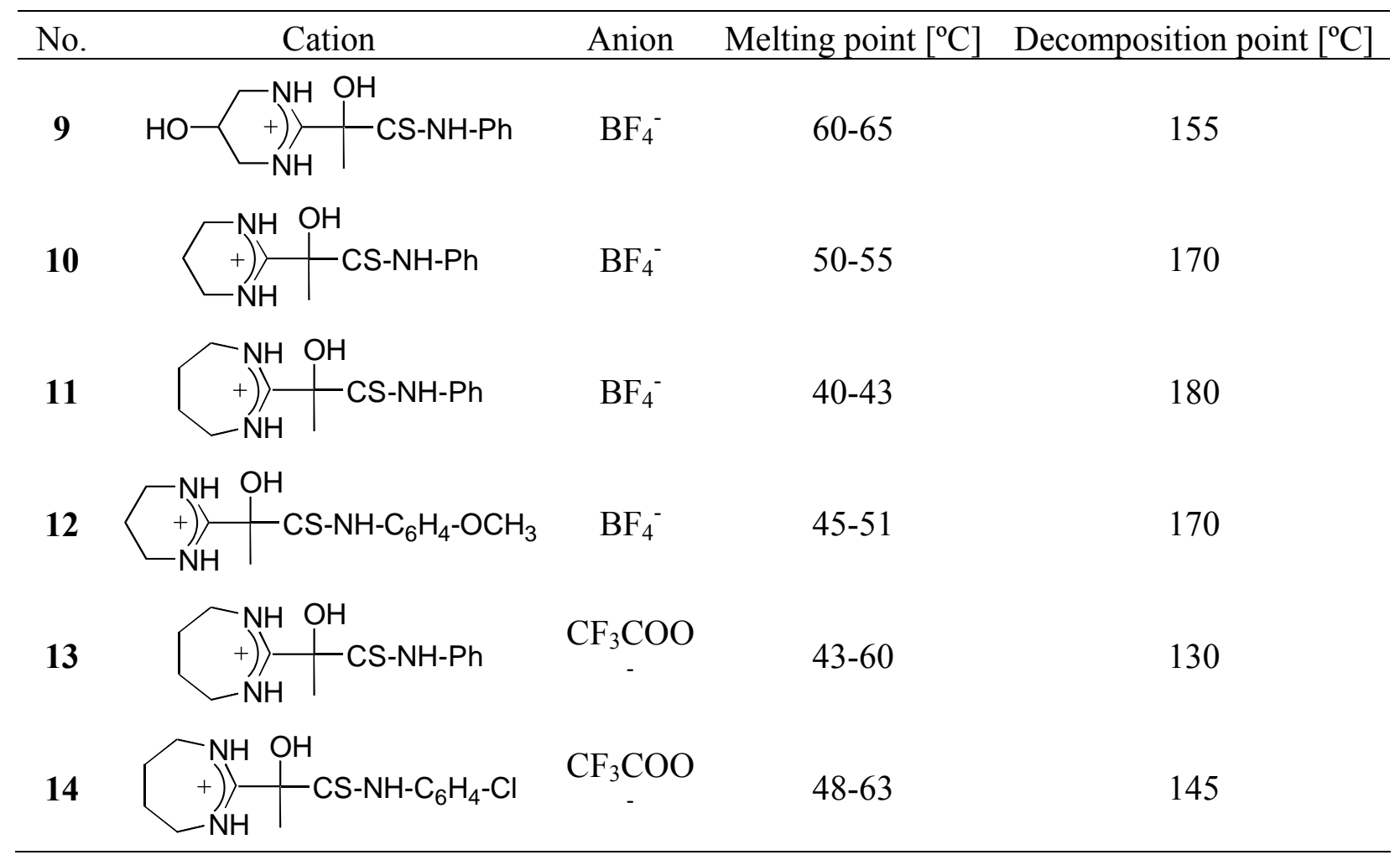

Above the temperatures given as decomposition point the salts start to darken. At room temperature they are pale yellow soft resinous materials soluble in water and alcohols. These compounds are sensitive to alkaline conditions and decompose to the starting zwitterionic substrates when treated with bases.

We made preliminary attempts to verify if salts of this type can serve as ionic media for some simple reactions and if there is a significant influence on their courses.

Since reactions of anilides and pyridilides of some 3-oxoacids were one of the major aspects of our recent works, ${ }^{22}$ we selected one of them as a reference reaction (Scheme 3). It has been shown $^{22}$ that reaction of pyridilide 15 with nitrosobenzene leads to formation of pyrido[1,2a][1,3,5]triazin-2-one $\mathbf{1 6}$ as the main product. Ionic liquid $\mathbf{1 0}$ was chosen as a medium for the reaction and reaction time was arbitrarily chosen as shorter than typical. We also decided to 
eliminate the catalyst normally used in the process. Comparison of reaction details is given in Table 2.<smiles>O=C(CC(=O)Nc1ccccn1)Nc1ccccc1</smiles>

15<smiles>O=c1nc2ccccn2c(=Nc2ccccc2)n1-c1ccccc1</smiles>

16<smiles>c1ccc(N=C2N(c3ccccc3)C(=Nc3ccccc3)N2c2ccccc2)cc1</smiles>

17

Scheme 3

Table 2. Details of the reaction in scheme 3

\begin{tabular}{cccccc}
\hline Reaction medium & Catalyst & Temp. $\left[{ }^{\circ} \mathrm{C}\right]$ & Time $[\mathrm{min}]$ & Yield of $\mathbf{1 6}$ & $\mathbf{1 6 : 1 7}$ ratio \\
\hline $\mathrm{MeOH}$ & $25 \% \mathrm{NaOH}$ & 25 & 120 & $52 \%$ & $2.4: 1$ \\
$\mathbf{1 0}$ & none & 70 & 20 & $35 \%$ & $4.5: 1$ \\
\hline
\end{tabular}

Reaction in ionic liquid $\mathbf{1 0}$ can give comparable yield of the main product in a significantly shorter time than in methanol. The use of a catalyst appeared to be unnecessary. The reaction seems to be directed to the formation of the product $\mathbf{1 6}$ and allows one to reduce the amount of the minor by-product 17. Isolation of the products can simply be done by addition of water and precipitation of compound $\mathbf{1 6}$ and subsequent extraction of aqueous phase with diethyl ether, which gives the solution of pure compound 17. Ionic liquid can then be fully recovered from the aqueous solution.

Other reactions were conducted using the same salt 10, like the Knoevenagel condensation, Diels-Alder reactions and simple esterifications, however products were obtained in low yields. In these cases the use of salt $\mathbf{1 0}$ appears to be problematic since side reactions are observed and this requires further investigations.

\section{Conclusions}

We investigated salts of a unique type with perhydropyrimidinylium, 1,3-diazepinylium and quinazolinylium carbocations and presented some of them which are low-melting. The initial attempts show that perhydropyrimidinylium salt with tetrafluoroborate anion $\mathbf{1 0}$ and probably other salts of this type may be utilized as reaction media and may have interesting effect on their route.

Zwitterionic compounds of the presented type are useful starting materials for in situ composing of various low-melting salts since it only requires simple treatment with appropriate 
acid. Because of the presence of the chiral centre in the cation, separation of enantiomers would furnish interesting ionic solvents for stereoselective processes.

\section{Experimental Section}

General Procedures. Melting points were determined on an electrothermal IA9000 digital melting point apparatus and are uncorrected. The IR spectra were obtained on a Bruker IFS 48 spectrometer at room temperature. ${ }^{1} \mathrm{H}$ and ${ }^{13} \mathrm{C}$ NMR spectra were recorded with a $300 \mathrm{NMR}$ spectrometer using TMS as internal standard. Chemical shifts are reported in ppm downfield from TMS.

Preparation, characteristics and spectral data for compounds $\mathbf{4 a}$ and $\mathbf{4 b}$ were already presented. ${ }^{23}$ Formation of salts appeared to be a key step in their isolation from reaction mixture and was a significant improvement in their purification. The reaction mixture was treated with hydrochloric acid and after evaporation to dryness the remaining residue was dissolved in methylene chloride. After standing crystals of salts of $\mathbf{4 a}$ and $\mathbf{4 b}$ were formed which could be crystallized from acetonitrile and after treatment with stoichiometric amount of morpholine gave pure zwitterions.

\section{General Procedure for the Preparation of compounds 5-8}

A solution of $2 \mathrm{~g}$ of anilide 1a, $1 \mathbf{b}$ or $1 \mathbf{c}(6.36 \mathrm{mmol}, 5.73 \mathrm{mmol}, 5.81 \mathrm{mmol}$ respectively $)$ and double of stoichiometric amount of a corresponding diamine in toluene was heated for about 1 hour in $85{ }^{\circ} \mathrm{C}$. In case of compounds $\mathbf{5 a}$ and $\mathbf{5 b}$ dihydrochloride of meso-2,4-pentanediamine was used with addition of stoichiometric amount of potassium tert-butoxide.

Isolation of compounds 5 and 8. The reaction mixture was then partially evaporated and cooled in ice bath. Colorless crystals of the product were formed which were washed with ethanol-ether mixture and crystallized from ethanol.

Isolation of compounds 6 and 7. The reaction mixture was evaporated and the residue treated with hydrochloric acid and the resulting yellow solid was washed with hot acetone. Next the solid was dissolved in water and treated with triethylamine in a small excess. After removing of solvent the remaining oil was solidified by treatment with diethyl ether/ethanol mixture and purified by recrystallization from ethanol.

4'R,6'S-2-Hydroxy-2-(4',6'-dimethylperhydropyrimidin-2'-ylium-2'-yl)-1-(phenylimino)propane-1-thiolate (5a). Yield $60 \%$; colorless crystals, mp 166-168 ${ }^{\circ} \mathrm{C}$; IR (KBr): $3261(\mathrm{NH})$, 2976, $1648(\mathrm{~N}-\mathrm{C}-\mathrm{N}), 1606,\left(\mathrm{C}=\mathrm{C}\right.$ aromatic) $\mathrm{cm}^{-1}$; ${ }^{1} \mathrm{H}-\mathrm{NMR}\left(\mathrm{DMSO}-d_{6}\right): \delta 8.56(\mathrm{~s}, 2 \mathrm{H}, \mathrm{NH}), 7.24$ (t, 2H, Ph, $J=9.0 \mathrm{~Hz}), 7.08$ (d, 2H, Ph, $J=9.0 \mathrm{~Hz}$ ), 6.93 (t, 1H, Ph, $J=9.0 \mathrm{~Hz}), 3.66-3.56$ (m, $2 \mathrm{H}, 2 \times \mathrm{N}-\mathrm{CH}), 2.15-2.08\left(\mathrm{~m}, 1 \mathrm{H}, \mathrm{CH}_{2}\right), 1.65\left(\mathrm{~s}, 3 \mathrm{H}, \mathrm{CH}_{3}\right), 1.26\left(\mathrm{~d}, 6 \mathrm{H}, 2 \times \mathrm{CH}_{3}\right), 1.21-1.09(\mathrm{~m}$, $\left.1 \mathrm{H}, \mathrm{CH}_{2}\right) ;{ }^{13} \mathrm{C}-\mathrm{NMR}\left(\mathrm{DMSO}-d_{6}\right): \delta 185.9(\mathrm{C}-\mathrm{S}), 166.0(\mathrm{~N}-\mathrm{C}-\mathrm{N}), 152.1,127.8,121.9(\mathrm{Ph}), 74.1$ $(\mathrm{C}-\mathrm{O}), 45.7(\mathrm{~N}-\mathrm{CH}), 45.5(\mathrm{~N}-\mathrm{CH}), 35.4\left(\mathrm{CH}_{3}\right), 29.6\left(\mathrm{CH}_{2}\right), 19.8\left(\mathrm{CH}_{3}\right)$. Anal. Calcd for $\mathrm{C}_{15} \mathrm{H}_{20} \mathrm{~N}_{3} \mathrm{OS}$ (291.4): C, $61.82 ; \mathrm{H}, 7.26$; N, 14.42. Found: C, $61.70 ; \mathrm{H}, 7.28 ; \mathrm{N}, 14.40$. 
4'R,6'S-2-Hydroxy-2-(4',6'-dimethylperhydropyrimidin-2'-ylium-2'-yl)-1-(4-chlorophenylimino)propane-1-thiolate (5b). Yield $72 \%$; colorless crystals, mp 189-190 ${ }^{\circ} \mathrm{C}$; IR $(\mathrm{KBr}): 3283(\mathrm{NH}), 2976,1645(\mathrm{~N}-\mathrm{C}-\mathrm{N}), 1601,\left(\mathrm{C}=\mathrm{C}\right.$ aromatic) $\mathrm{cm}^{-1} ;{ }^{1} \mathrm{H}-\mathrm{NMR}\left(\mathrm{DMSO}-d_{6}\right): \delta$ 8.45 (s, 2H, NH), 7.26 (d, 2H, Ph, $J=9.0 \mathrm{~Hz}$ ), 7.10 (d, 2H, Ph, $J=9.0 \mathrm{~Hz}$ ), 3.67-3.56 (m, 2H, 2 x N-CH), 2.15-2.07 (m, 1H, $\left.\mathrm{CH}_{2}\right), 1.65\left(\mathrm{~s}, 3 \mathrm{H}, \mathrm{CH}_{3}\right), 1.27\left(\mathrm{~d}, 6 \mathrm{H}, 2 \mathrm{x} \mathrm{CH}_{3}\right), 1.21-1.09(\mathrm{~m}, 1 \mathrm{H}$, $\left.\mathrm{CH}_{2}\right) ;{ }^{13} \mathrm{C}-\mathrm{NMR}\left(\mathrm{DMSO}-d_{6}\right): \delta 186.9(\mathrm{C}-\mathrm{S}), 165.9(\mathrm{~N}-\mathrm{C}-\mathrm{N}), 151.2,127.7,125.5,123.7,(\mathrm{Ph})$, $74.4(\mathrm{C}-\mathrm{O}), 45.7(\mathrm{~N}-\mathrm{CH}), 45.5(\mathrm{~N}-\mathrm{CH}), 35.4\left(\mathrm{CH}_{3}\right), 29.1\left(\mathrm{CH}_{2}\right), 19.8\left(\mathrm{CH}_{3}\right)$. Anal. Calcd for $\mathrm{C}_{15} \mathrm{H}_{20} \mathrm{ClN}_{3} \mathrm{OS}$ (325.9): C, 55.29 ; H, 6.19; N, 12.90. Found: C, 55.25 ; H, 6.00 ; N, 13.00.

2-Hydroxy-2-(4'-ethylperhydropyrimidin-2'-ylium-2'-yl)-1-(phenylimino)propane-1-

thiolate (6a). Yield $55 \%$; colorless crystals, mp 143-144 ${ }^{\circ} \mathrm{C}$; IR (KBr): 3250 (NH), 2987, 1661 $(\mathrm{N}-\mathrm{C}-\mathrm{N}), 1597,\left(\mathrm{C}=\mathrm{C}\right.$ aromatic) $\mathrm{cm}^{-1} ;{ }^{1} \mathrm{H}-\mathrm{NMR}\left(\mathrm{DMSO}-d_{6}\right): \delta 8.53(\mathrm{~s}, 2 \mathrm{H}, \mathrm{NH}), 7.35(\mathrm{~d}, 2 \mathrm{H}, \mathrm{Ph}$, $J=9.0 \mathrm{~Hz}), 6.92(\mathrm{t}, 2 \mathrm{H}, \mathrm{Ph}, J=9.0 \mathrm{~Hz}), 6.81(\mathrm{t}, 1 \mathrm{H}, \mathrm{Ph}, J=9.0 \mathrm{~Hz}), 3.52-3.41(\mathrm{~m}, 1 \mathrm{H}, \mathrm{N}-\mathrm{CH})$, 3.40-3.32 (m, 2H, N-CH $), 2.10\left(\mathrm{~s}, 3 \mathrm{H}, \mathrm{CH}_{3}\right), 1.90-1.60(\mathrm{~m}, 4 \mathrm{H}), 1.05\left(\mathrm{t}, 3 \mathrm{H}, \mathrm{CH}_{3}\right) ;{ }^{13} \mathrm{C}-\mathrm{NMR}$ (DMSO- $\left.d_{6}\right): \delta 186.4(\mathrm{C}-\mathrm{S}), 165.2(\mathrm{~N}-\mathrm{C}-\mathrm{N}), 151.4,126.1,124.9,122.1(\mathrm{Ph}), 75.1(\mathrm{C}-\mathrm{O}), 51.8(\mathrm{~N}-$ $\mathrm{CH}), 38.3\left(\mathrm{~N}_{\left.-\mathrm{CH}_{2}\right)}\right), 28.7\left(\mathrm{CH}_{3}\right), 27.9\left(\mathrm{CH}_{2}\right), 23.2,\left(\mathrm{CH}_{2}\right), 9.8\left(\mathrm{CH}_{3}\right)$. Anal. Calcd for $\mathrm{C}_{15} \mathrm{H}_{21} \mathrm{~N}_{3} \mathrm{OS}$ (291.4): C, $61.82 ; \mathrm{H}, 7.26$; N, 14.42. Found: C, $61.75 ; \mathrm{H}, 7.30$; N, 14.38.

2-Hydroxy-2-(4'-ethylperhydropyrimidin-2'-ylium-2'-yl)-1-(4-chlorophenylimino)propane1-thiolate (6b). Yield $59 \%$; colorless crystals, mp 151-152 ${ }^{\circ} \mathrm{C}$; IR (KBr): 3262 (NH), 2989, $1658(\mathrm{~N}-\mathrm{C}-\mathrm{N}), 1592,\left(\mathrm{C}=\mathrm{C}\right.$ aromatic) $\mathrm{cm}^{-1} ;{ }^{1} \mathrm{H}-\mathrm{NMR}\left(\mathrm{DMSO}-d_{6}\right): \delta 8.56(\mathrm{~s}, 2 \mathrm{H}, \mathrm{NH}), 7.30(\mathrm{~d}$, $2 \mathrm{H}, \mathrm{Ph}, J=9.0 \mathrm{~Hz}), 7.00(\mathrm{~d}, 2 \mathrm{H}, \mathrm{Ph}, J=9.0 \mathrm{~Hz}), 3.56-3.44(\mathrm{~m}, 1 \mathrm{H}, \mathrm{N}-\mathrm{CH}), 3.44-3.31(\mathrm{~m}, 2 \mathrm{H}$, $\left.\mathrm{N}-\mathrm{CH}_{2}\right), 2.07\left(\mathrm{~s}, 3 \mathrm{H}, \mathrm{CH}_{3}\right), 1.88-1.39(\mathrm{~m}, 4 \mathrm{H}), 0.87\left(\mathrm{t}, 3 \mathrm{H}, \mathrm{CH}_{3}\right) ;{ }^{13} \mathrm{C}-\mathrm{NMR}\left(\mathrm{DMSO}-d_{6}\right): \delta 186.4$ (C-S), $165.2(\mathrm{~N}-\mathrm{C}-\mathrm{N}), 151.4,126.1,124.9,122.1(\mathrm{Ph}), 74.31(\mathrm{C}-\mathrm{O}), 48.9(\mathrm{~N}-\mathrm{CH}), 36.5\left(\mathrm{~N}_{-} \mathrm{CH}_{2}\right)$, $26.5\left(\mathrm{CH}_{3}\right), 25.6\left(\mathrm{CH}_{2}\right), 21.2,\left(\mathrm{CH}_{2}\right), 9.3\left(\mathrm{CH}_{3}\right)$. Anal. Calcd for $\mathrm{C}_{15} \mathrm{H}_{20} \mathrm{~N}_{3} \mathrm{OS}(300.8)$ : C, 51.90 ; H, 6.37 ; N, 13.97. Found: C, $51.85 ; \mathrm{H}, 6.31 ; \mathrm{N}, 14.07$.

2-Hydroxy-2-(perhydropyrimidin-2'-ylium-2'-yl)-1-(4-methoxyphenylimino)-propane-1thiolate (2c). Yield $57 \%$; colorless crystals, mp 122-123 ${ }^{\circ} \mathrm{C}$; IR (KBr): $3302(\mathrm{NH}), 2927,1655$ $(\mathrm{N}-\mathrm{C}-\mathrm{N}), 1592,\left(\mathrm{C}=\mathrm{C}\right.$ aromatic) $\mathrm{cm}^{-1} ;{ }^{1} \mathrm{H}-\mathrm{NMR}\left(\mathrm{DMSO}-d_{6}\right): \delta 8.60(\mathrm{~s}, 2 \mathrm{H}, \mathrm{NH}), 7.20(\mathrm{~d}, 2 \mathrm{H}, \mathrm{Ph}$, $J=8.5 \mathrm{~Hz}), 6.80(\mathrm{~d}, 2 \mathrm{H}, \mathrm{Ph}, J=8.5 \mathrm{~Hz}), 3.71\left(\mathrm{~s}, 3 \mathrm{H}, \mathrm{CH}_{3} \mathrm{O}\right), 3.36-3.32\left(\mathrm{~m}, 4 \mathrm{H}, 2 \times \mathrm{N}-\mathrm{CH}_{2}\right)$, 1.83-1.75 (m, 2H, $\left.\mathrm{CH}_{2}\right), 1.60\left(\mathrm{~s}, 3 \mathrm{H}, \mathrm{CH}_{3}\right) ;{ }^{13} \mathrm{C}-\mathrm{NMR}\left(\mathrm{DMSO}-d_{6}\right): \delta 183.8(\mathrm{C}-\mathrm{S}), 166.4(\mathrm{~N}-\mathrm{C}-$ $\mathrm{N})$, 154.5, 145.1, 123.3, 112.9, $(\mathrm{Ph}), 74.7(\mathrm{C}-\mathrm{O}), 55.0\left(\mathrm{CH}_{3} \mathrm{O}\right), 38.4\left(\mathrm{~N}_{-} \mathrm{CH}_{2}\right), 28.8\left(\mathrm{CH}_{3}\right), 17.8$ $\left(\mathrm{CH}_{2}\right)$. Anal. Calcd for $\mathrm{C}_{15} \mathrm{H}_{20} \mathrm{~N}_{3} \mathrm{OS}$ (279.4): C, 55.90 ; H, 6.13 ; N, 15.04. Found: C, 55.90 ; H, $5.88 ; \mathrm{N}, 15.09$.

2-Hydroxy-2-(5-Hydroxyperhydropyrimidin-2'-ylium-2'-yl)-1-(phenylimino)-propane-1thiolate (7). Yield $41 \%$; colorless crystals, mp 178-179 ${ }^{\circ} \mathrm{C}$; IR (KBr): $3353(\mathrm{NH}), 2989,1658$ $(\mathrm{N}-\mathrm{C}-\mathrm{N}), 1584,\left(\mathrm{C}=\mathrm{C}\right.$ aromatic) $\mathrm{cm}^{-1} ;{ }^{1} \mathrm{H}-\mathrm{NMR}\left(\mathrm{DMSO}-d_{6}\right): \delta 8.52(\mathrm{~s}, 2 \mathrm{H}, \mathrm{NH}), 7.21(\mathrm{t}, 2 \mathrm{H}, \mathrm{Ph}$, $J=7.5 \mathrm{~Hz}$ ), 7.05 (d, 2H, Ph, $J=7.5 \mathrm{~Hz}), 6.90$ (t, 1H, Ph, J=7.5 Hz), 4.09-4.05 (q, 1H, CH-O), 3.37-3.31 (m, 4H, $2 \times$ N-CH$), 1.62\left(\mathrm{~s}, 3 \mathrm{H}, \mathrm{CH}_{3}\right) ;{ }^{13} \mathrm{C}-\mathrm{NMR}\left(\mathrm{DMSO}-d_{6}\right): \delta 185.1(\mathrm{C}-\mathrm{S}), 165.9$ $(\mathrm{N}-\mathrm{C}-\mathrm{N}), 152.6,127.7,122.1,121.7,(\mathrm{Ph}), 74.6(\mathrm{C}-\mathrm{O}), 56.7(\mathrm{CH}-\mathrm{OH}), 49.5\left(\mathrm{~N}_{-} \mathrm{CH}_{2}\right), 27.6\left(\mathrm{CH}_{3}\right)$. Anal. Calcd for $\mathrm{C}_{15} \mathrm{H}_{20} \mathrm{~N}_{3} \mathrm{OS}$ (279.4): C, 55.90 ; H, 6.13 ; N, 15.04. Found: C, 55.90 ; H, 5.88 ; N, 15.09. 
2-Hydroxy-2-(5',5'-Dimethylperhydropyrimidin-2'-ylium-2'-yl)-1-(phenylimino)-pro-pane1-thiolate (8). Yield $64 \%$; colorless crystals, mp 159-160 ${ }^{\circ} \mathrm{C}$; IR (KBr): 3368 (NH), 2926, 1651 $(\mathrm{N}-\mathrm{C}-\mathrm{N}), 1584,\left(\mathrm{C}=\mathrm{C}\right.$ aromatic) $\mathrm{cm}^{-1} ;{ }^{1} \mathrm{H}-\mathrm{NMR}\left(\mathrm{DMSO}-d_{6}\right): \delta 8.55(\mathrm{~s}, 2 \mathrm{H}, \mathrm{NH}), 7.20(\mathrm{t}, 2 \mathrm{H}, \mathrm{Ph}$, $J=7.5 \mathrm{~Hz}), 6.94$ (d, 2H, Ph, $J=7.5 \mathrm{~Hz}), 6.87$ (t, 1H, Ph, $J=7.5 \mathrm{~Hz}), 3.07-3.00$ (m, 4H, 2 x N$\left.\mathrm{CH}_{2}\right), 1.63\left(\mathrm{~s}, 3 \mathrm{H}, \mathrm{CH}_{3}\right), 0.94\left(\mathrm{~s}, 6 \mathrm{H}, 2 \times \mathrm{CH}_{3}\right) ;{ }^{13} \mathrm{C}-\mathrm{NMR}\left(\mathrm{DMSO}-d_{6}\right): \delta 185.0(\mathrm{C}-\mathrm{S}), 165.6(\mathrm{~N}-$ C-N), 153.2, 127.8, 121.8, 121.5, (Ph), $74.9(\mathrm{C}-\mathrm{O}), 49.5\left(\mathrm{~N}-\mathrm{CH}_{2}\right), 27.6\left(\mathrm{CH}_{3}\right), 25.21\left(\mathrm{CMe}_{2}\right)$, $23.2\left(\mathrm{CH}_{3}\right)$. Anal. Calcd for $\mathrm{C}_{15} \mathrm{H}_{20} \mathrm{~N}_{3} \mathrm{OS}$ (291.4): C, 61.83 ; H, 7.26 ; N, 14.42. Found: C, 61.89 ; H, 7.32 ; N, 14.54.

2-(1'-Phenylthiocarbamoyl-1'-hydroxyethyl)-5-hydroxyperhydropyrimidin-2-ylium tetrafluoroborate (9). Yellow glass, $\mathrm{mp} 60-65^{\circ} \mathrm{C}$; IR (KBr): 3280 ( $\mathrm{NH}$ thioamide), 2900, 1650 $(\mathrm{N}-\mathrm{C}-\mathrm{N}), 1590\left(\mathrm{C}=\mathrm{C}\right.$ aromatic), $1050(\mathrm{~B}-\mathrm{F}) \mathrm{cm}^{-1} ;{ }^{1} \mathrm{H}-\mathrm{NMR}$ (DMSO- $\left.d_{6}\right): \delta 11.37(\mathrm{~s}, 1 \mathrm{H}, \mathrm{NH}$, thioamide), 9.32 (s, 2H, NH), $8.01(\mathrm{~s}, 1 \mathrm{H}, \mathrm{OH}), 7.70$ (d, 2H, Ph, J=7.5 Hz), 7.45 (t, 2H, Ph, J= $7.5 \mathrm{~Hz}$ ), 7.30 (t, 1H, Ph, J= 7.5), 5.21 (s, broad, CH-OH), 4.13 (m, CH-OH), 3.44-3.39 (m, 4H, $\left.2 \times \mathrm{N}-\mathrm{CH}_{2}\right), 1.87\left(\mathrm{~s}, 3 \mathrm{H}, \mathrm{CH}_{3}\right) ;{ }^{13} \mathrm{C}-\mathrm{NMR}\left(\mathrm{DMSO}-d_{6}\right): \delta 197.9(\mathrm{C}=\mathrm{S}), 163.1(\mathrm{~N}-\mathrm{C}-\mathrm{N}), 138.3$, 128.5, 126.9, $124.3(\mathrm{Ph}), 79.6(\mathrm{C}-\mathrm{O}), 56.3(\mathrm{HC}-\mathrm{O}), 45.0\left(\mathrm{~N}-\mathrm{CH}_{2}\right), 26.0\left(\mathrm{CH}_{3}\right)$. Anal. Calcd for $\mathrm{C}_{13} \mathrm{H}_{18} \mathrm{~N}_{3} \mathrm{O}_{2} \mathrm{BSF}_{4}$ (367.2): C, 42.53 ; H, 4.94 ; N, 11.44. Found: C, 42.46 ; H, 5.07 ; N, 11.50.

2-(1'-Phenylthiocarbamoyl-1'-hydroxyethyl)perhydropyrimidin-2-ylium tetrafluoroborate (10). Yellow glass, mp 50-55 ${ }^{\circ} \mathrm{C}$; IR (KBr): 3250 (NH thioamide), 2930, 1650 (N-C-N), 1590 $\left(\mathrm{C}=\mathrm{C}\right.$ aromatic), $1052(\mathrm{~B}-\mathrm{F}) \mathrm{cm}^{-1} ;{ }^{1} \mathrm{H}-\mathrm{NMR}\left(\mathrm{DMSO}-d_{6}\right): \delta 11.41(\mathrm{~s}, 1 \mathrm{H}, \mathrm{NH}$, thioamide), $9.41(\mathrm{~s}$, 2H, NH), 7.96 (s, 1H, OH), 7.72 (d, 2H, Ph, J=7.5 Hz), 7.47 (t, 2H, Ph, J=7.5 Hz), 7.32 (t, 1H, $\mathrm{Ph}, J=7.5)$, 3.44-3.38 (m, 4H, $\left.2 \times \mathrm{N}-\mathrm{CH}_{2}\right), 1.87\left(\mathrm{~s}, 3 \mathrm{H}, \mathrm{CH}_{3}\right), 1.84\left(\mathrm{~m}, 2 \mathrm{H}, \mathrm{CH}_{2}\right) ;{ }^{13} \mathrm{C}-\mathrm{NMR}$ $\left(\mathrm{DMSO}-d_{6}\right): \delta 198.0(\mathrm{C}=\mathrm{S}), 163.7(\mathrm{~N}-\mathrm{C}-\mathrm{N}), 138.3,128.5,126.9,124.3(\mathrm{Ph}), 79.8(\mathrm{C}-\mathrm{O}), 38.7$ $\left(\mathrm{N}-\mathrm{CH}_{2}\right), 25.9\left(\mathrm{CH}_{3}\right), 17.3\left(\mathrm{CH}_{2}\right)$. Anal. Calcd for $\mathrm{C}_{13} \mathrm{H}_{18} \mathrm{~N}_{3} \mathrm{OBSF}_{4}$ (351.2): C, $44.46 ; \mathrm{H}, 5.17$; N, 11.97. Found: C, $44.51 ; \mathrm{H}, 5.02 ; \mathrm{N}, 12.09$.

2-(1'-Phenylthiocarbamoyl-1'-hydroxyethyl)perhydrodiazepin-2-ylium tetrafluoroborate (11). Yellow glass, mp 40-43 ${ }^{\circ} \mathrm{C}$; IR (KBr): 3250 (NH thioamide), 2920, 1650 (N-C-N), 1590 $\left(\mathrm{C}=\mathrm{C}\right.$ aromatic), $1050(\mathrm{~B}-\mathrm{F}) \mathrm{cm}^{-1} ;{ }^{1} \mathrm{H}-\mathrm{NMR}\left(\mathrm{DMSO}-d_{6}\right): \delta 11.38(\mathrm{~s}, 1 \mathrm{H}, \mathrm{NH}$, thioamide), $9.10(\mathrm{~s}$, 2H, NH), 8.00 (s, 1H, OH), $7.72(\mathrm{~d}, 2 \mathrm{H}, \mathrm{Ph}, J=7.5 \mathrm{~Hz}), 7.46$ (t, 2H, Ph, J=7.5 Hz), 7.32 (t, 1H, $\mathrm{Ph}, J=7.5), 3.48-3.40\left(\mathrm{~m}, 4 \mathrm{H}, 2 \times \mathrm{N}-\mathrm{CH}_{2}\right), 1.95\left(\mathrm{~m}, 4 \mathrm{H}, 2 \times \mathrm{CH}_{2}\right), 1.86\left(\mathrm{~s}, 3 \mathrm{H}, \mathrm{CH}_{3}\right) ;{ }^{13} \mathrm{C}-\mathrm{NMR}$ $\left(\mathrm{DMSO}-d_{6}\right): \delta 198.0(\mathrm{C}=\mathrm{S}), 168.8(\mathrm{~N}-\mathrm{C}-\mathrm{N}), 138.3,128.5,126.9,124.3(\mathrm{Ph}), 80.7(\mathrm{C}-\mathrm{O}), 42.7$ $\left(\mathrm{N}-\mathrm{CH}_{2}\right), 25.9\left(\mathrm{CH}_{3}\right), 25.1\left(\mathrm{CH}_{2}\right)$. Anal. Calcd for $\mathrm{C}_{14} \mathrm{H}_{20} \mathrm{~N}_{3} \mathrm{OBSF}_{4}(365.2)$ : C, $46.04 ; \mathrm{H}, 5.52$; N, 11.51. Found: C, $46.10 ; \mathrm{H}, 5.42 ; \mathrm{N}, 11.52$.

\section{2-(1'-(4-Methoxyphenyl)thiocarbamoyl-1'-hydroxyethyl)perhydropyrimidin-2-ylium}

tetrafluoroborate (12). Yellow glass, $\mathrm{mp} 45-51^{\circ} \mathrm{C}$; IR (KBr): 3250 (NH thioamide), 2940, 1650 $(\mathrm{N}-\mathrm{C}-\mathrm{N}), 1590\left(\mathrm{C}=\mathrm{C}\right.$ aromatic), $1050(\mathrm{~B}-\mathrm{F}) \mathrm{cm}^{-1}$; ${ }^{1} \mathrm{H}-\mathrm{NMR}\left(\mathrm{DMSO}-d_{6}\right): \delta 11.29(\mathrm{~s}, 1 \mathrm{H}, \mathrm{NH}$, thioamide), 9.36 (s, 2H, NH), $7.88(\mathrm{~s}, 1 \mathrm{H}, \mathrm{OH}), 7.62$ (d, 2H, Ph, J=8.7 Hz), 6.98 (d, 2H, Ph, J= $8.7 \mathrm{~Hz}), 3.77\left(\mathrm{~s}, 3 \mathrm{H}, \mathrm{CH}_{3} \mathrm{O}\right), 3.40-3.35\left(\mathrm{~m}, 4 \mathrm{H}, 2 \times \mathrm{N}_{-} \mathrm{CH}_{2}\right), 1.83\left(\mathrm{~s}, 3 \mathrm{H}, \mathrm{CH}_{3}\right), 1.80(\mathrm{~m}, 2 \mathrm{H}$, $\left.\mathrm{CH}_{2}\right) ;{ }^{13} \mathrm{C}-\mathrm{NMR}\left(\mathrm{DMSO}-d_{6}\right): \delta 197.1(\mathrm{C}=\mathrm{S}), 163.8(\mathrm{~N}-\mathrm{C}-\mathrm{N}), 157.6,131.2,125.6,113.6(\mathrm{Ph})$, 79.7 (C-O), $55.25\left(\mathrm{CH}_{3} \mathrm{O}\right), 38.7\left(\mathrm{~N}-\mathrm{CH}_{2}\right), 25.9\left(\mathrm{CH}_{3}\right), 17.3\left(\mathrm{CH}_{2}\right)$. Anal. Calcd for $\mathrm{C}_{14} \mathrm{H}_{20} \mathrm{~N}_{3} \mathrm{O}_{2} \mathrm{BSF}_{4}$ (381.2): C, $44.11 ; \mathrm{H}, 5.29$; N, 11.02. Found: C, 44.20 ; H, 5.31 ; N, 12.98. 
2-(1'-Phenylthiocarbamoyl-1'-hydroxyethyl)perhydro-1,3-diazepin-2-ylium trifluoroacetate (13). Yellow glass, mp 43-60 ${ }^{\circ} \mathrm{C}$; IR (KBr): $3420\left(\mathrm{NH}\right.$ thioamide), $1179(\mathrm{C}=\mathrm{S}) \mathrm{cm}^{-1}$; ${ }^{1} \mathrm{H}-\mathrm{NMR}$ (DMSO-d $)_{6}$ ): 11.38 (s, 1H, NH, thioamide), 9.10 (s, 2H, NH), $8.22(\mathrm{~s}, 1 \mathrm{H}, \mathrm{OH}), 7.73$ (d, 2H, Ph, $J=8.0 \mathrm{~Hz}$ ), 7.44 (t, 2H, Ph, $J=8.0 \mathrm{~Hz}), 7.31$ (t, 1H, Ph, $J=8.0), 3.65-3.56\left(\mathrm{~m}, 4 \mathrm{H}, 2 \mathrm{~N}_{-} \mathrm{CH}_{2}\right.$ ), 2.00-1.89 (m, 4H, C-CH$\left.-\mathrm{CH}_{2}-\mathrm{C}\right), 1.86\left(\mathrm{~s}, 3 \mathrm{H}, \mathrm{CH}_{3}\right) ;{ }^{13} \mathrm{C}-\mathrm{NMR}\left(\mathrm{DMSO}-d_{6}\right): \delta 198.0(\mathrm{C}=\mathrm{S})$, $168.8(\mathrm{~N}-\mathrm{C}-\mathrm{N}), 138.3,128.4,126.7,124.1(\mathrm{Ph}), 80.6(\mathrm{C}-\mathrm{O}), 42.7\left(\mathrm{~N}_{-} \mathrm{CH}_{2}\right), 25.8\left(\mathrm{CH}_{3}\right), 25.1$ $\left(\mathrm{CH}_{2}\right)$. Anal. Calcd for $\mathrm{C}_{16} \mathrm{H}_{20} \mathrm{~N}_{3} \mathrm{O}_{3} \mathrm{SF}_{3}$ (391.4): C, 49.10 ; H, 5.15 ; N, 10.74. Found: C, 48.93 ; $\mathrm{H}, 5.21 ; \mathrm{N}, 10.65$.

2-(1'-p-Chlorophenylthiocarbamoyl-1'-hydroxyethyl)perhydro-1,3-diazepin-2-ylium trifluoroacetate (14). Yellow glass, mp 48-63 ${ }^{\circ} \mathrm{C}$; IR ( $\left.\mathrm{KBr}\right): 3420$ (NH thioamide), $1178(\mathrm{C}=\mathrm{S}) \mathrm{cm}^{-1}$; ${ }^{1} \mathrm{H}-\mathrm{NMR}$ (DMSO-d $\left.d_{6}\right): \delta 11.48(\mathrm{~s}, 1 \mathrm{H}, \mathrm{NH}$, thioamide), $9.10(\mathrm{~s}, 2 \mathrm{H}, \mathrm{NH}), 8.22(\mathrm{~s}, 1 \mathrm{H}, \mathrm{OH}), 7.75(\mathrm{~d}, 2 \mathrm{H}, \mathrm{Ph}$, $J=9.0 \mathrm{~Hz}$ ), $7.50(\mathrm{~d}, 2 \mathrm{H}, \mathrm{Ph}, J=9.0 \mathrm{~Hz}), 3.65-3.55$ (m, 4H, $\left.2 \times \mathrm{N}-\mathrm{CH}_{2}\right), 2.00-1.85$ (m, 4H, C$\left.\mathrm{CH}_{2}-\mathrm{CH}_{2}-\mathrm{C}\right), 1.85\left(\mathrm{~s}, 3 \mathrm{H}, \mathrm{CH}_{3}\right) ;{ }^{13} \mathrm{C}-\mathrm{NMR}$ (DMSO-d $): \delta 198.6(\mathrm{C}=\mathrm{S}), 168.7(\mathrm{~N}-\mathrm{C}-\mathrm{N}), 137.3$, 130.6, 128.4, $126.0(\mathrm{Ph}), 80.7(\mathrm{C}-\mathrm{O}), 42.7\left(\mathrm{~N}^{\left.-\mathrm{CH}_{2}\right),} 25.8\left(\mathrm{CH}_{3}\right), 25.1\left(\mathrm{CH}_{2}\right)\right.$. Anal. Calcd for $\mathrm{C}_{16} \mathrm{H}_{19} \mathrm{~N}_{3} \mathrm{O}_{3} \mathrm{SClF}_{3}$ (425.9): C, 45.13 ; H, 4.50 ; N, 9.87. Found: C, 44.98 ; H, 4.50 ; N, 9.77.

\section{References and Notes}

1. Gordon, C. M. Appl. Catal., A 2001, 222, 101.

2. Welton, T. Coord. Chem. Rev. 2004, 248, 2459.

3. Qiao, K.; Hagiwara, H.; Yokoyama, C. J. Mol. Catal. A: Chem. 2006, 246, 65.

4. Zhao, D.; Wu, M.; Kou, Y.; Min, E. Catal. Today 2002, 74, 157.

5. Pfruender, H.; Jones, R.; Weuster-Botz, D. J. Biotech. 2006, 124, 182.

6. Yang, Z.; Pan, W. Enzyme Microbial Technol. 2005, 37, 19.

7. Ohno, H.; Yoshizawa, M.; Ogihara, W. Electrochim. Acta 2004, 50, 255.

8. Kubisa, P. Prog. Polym. Sci. 2004, 29, 3.

9. Hubbard, S. C.; Jones, P. B. Tetrahedron 2005, 61, 7425.

10. Suzuki, K.; Yamaguchi, M.; Kumagai, M.; Tanabe, N.; Yanagida S. C.R. Chimie 2006, 9, 611.

11. Usui, H.; Matsui, H.; Tanabe, N.; Yanagida, S. J. Photochem. Photobiol. A 2004, 164, 97.

12. Wang, H.; Lu, Q.; Ye C.; Liu, W.; Cui, Z. Wear 2004, 256, 44.

13. Lu, Q.; Wang, H.; Ye, C.; Liu, W.; Xue, Q. Tribol. Int. 2004, 37, 547.

14. Dai, S.; Yu, Y. H.; Gao, H. J.; Lin, J. S.; Pennycook, S. J.; Barnes, C. E. Chem. Comm. 2000, 243.

15. Xiaohua, X.; Liang, Z.; Xia, L.; Shengxiang, J. Anal. Chim. Acta 2004, 519, 207.

16. Majewski, P.; Pernak, A.; Grzymisławski, M.; Iwanik, K.; Pernak, J. Acta Histochem. 2003, 105(2), 135.

17. Zaleska, B.; Bazanek, T.; Socha, R.; Karelus, M.; Grochowski, J.; Serda, P. J. Org. Chem. 2002, 67, 4526. 
18. Zaleska, B.; Socha, R.; Karelus, M.; Szneler, E.; Grochowski, J.; Serda, P. J. Org. Chem. 2003, 68, 2334.

19. Sheldrick, G. M., 1997, SHELXS97 - Program for crystal structure solution, Univ. Göttingen, Germany.

20. Sheldrick, G. M., 1997, SHELXL97 - Program for crystal structure refinement, Univ. Göttingen, Germany.

21. Farrugia, L. J. J. Appl. Cryst. 1997, 32, 837.

22. Zaleska, B.; Trzewik, B.; Stodolak, E.; Grochowski, J.; Serda, P. Synthesis 2003, 16, 2559.

23. Zaleska, B.; Trzewik, B.; Karelus, M. Synthesis (in press). 